\title{
How to Select Development Language in Computer Software Development
}

\author{
Bin Shao, Xin Li \\ Langfang Polytechnic Institute, Langfang, 065000, China
}

\begin{abstract}
Keywords: Develop programming language; Computer software; $\mathrm{C}++$ language; JAVA programming language
\end{abstract}

\begin{abstract}
The $21^{\text {st }}$ century is a computer information technology era; with gradually quickening of speed of computer updating and more and more developed software industry, the computer industry proposes higher and higher development requirement for computer software program. In numerous applied structure and programming language with different performance, the reasonable selection can exert the high efficiency and high adaptability of software development to maximum degree. This paper carries out a discussion on several mainstream programming language in the industry at present, and then carries out analysis on applicability selection principle of development language in the computer software development process according to their features and specific application.
\end{abstract}

\section{Introduction}

So far, there are more than 30 kinds of programming language used for developing software program; they have different programming advantage and play an important role in actual software development project. While we select programming language, we shall get a deep understanding of features and functions of each language, start from perspective of actual project, and combine with the direction and property of developed software to select most suitable programming language.

\section{Mainstream programming language for software development}

\section{C language}

The $\mathrm{C}$ language is the bellwether in computer programming language, and it is widely applied and has excellent performance; it provides a kind of easiest way of program compilation, and it is especially effective for processing low-level memory as well as programming language which can cause small quantity of machine code or fully doesn't need the support of any operation environment.

As a kind of programming language with the widest scope of application in the world, C language almost involves in all software programs. In the 1980s, in order to avoid the difference caused by C language grammar on other programming language, American National Standards Institute (ANSI) especially set a set of international standard grammar ANSIC, which became the basic standard of C language. This set of standard basically considers high-level language and low-level language, and it can combine with basic structure of high-level and low-level language well. Therefore, $\mathrm{C}$ language successfully plays a multi-face role between high-level language and assembly language, thus it is also called "middle-level language".

Compared to other programming language, C language has great advantages. Firstly, it has 34 operators and has the strongest richness in language expression and strongest expressive ability among all programming language. Secondly, C language has 9 kinds of control statements and 32 key words. Therefore, while we use $\mathrm{C}$ language to carry out programming, we can make the language more tight and concise. As a whole, the mode of operation is flexible, and $\mathrm{C}$ language can meet the possible details in programming. Thirdly, $C$ language has large free space in design with few restraints and convenient use, and it has strong graphic function, which benefits from the situation that $\mathrm{C}$ language has strong ability of logic judgment and computing ability. Finally, $\mathrm{C}$ language also has the strongest universality among all programming language and it is applicable to most of software and system operation and development process, thus $\mathrm{C}$ language has good portability and wide application. 


\section{JAVA language}

JAVA is also a kind of programming development language which can span over platform and has wide scope of application, and it has high efficiency, security, and platform portability not inferior to $\mathrm{C}$ language. JAVA language can be seen in data center, game console, internet, mobile phone, and PC field.

In order to simplify the operation, JAVA programming language has no automatic enforcement measure and it adopts the way of multi-level inheritance and multi-angle computation, which simplifies the programming process. Due to strong independent technology support, while we use JAVA to carry out programming, we shall firstly carry out intermediate code compilation, and then install and check the code, and later explain the program coding. With wide application of computer software technology, this concept of programming thinking is widely accepted by people; therefore, in order to exert object-oriented advantage to maximum degree, the software program development personnel will carry out classification of objective elements for JAVA language to make its member variable and parameter packaging become more featured and explain the feature of elements so as to exert the function of explaining elements. While the new method and actual case are added into software development, JAVA can freely face them without influencing flexibility of language programming; on the contrary, the flexibility becomes more obvious. Besides, JAVA programming language can realize encryption technology, for the indicator in JAVA can produce different change to external world according to different situation. If any change happens in the indicator, JAVA can cut off the access to external data at the first time, which effectively avoids the situation that the data is destroyed or lost. Therefore, in the process of computer software development and application, there is a higher security in case of application of JAVA language ${ }^{[1]}$.

\section{Other programming language}

Except for above two universal programming language, $\mathrm{C}++$ is also a kind of mature, complicated, and popular programming design language. Under most condition, $\mathrm{C}++$ language is often applied in the design under Windows and Linux system environment. due to the fact hat $\mathrm{C}++$ code has executable program of computer binary code after compilation, $\mathrm{C}++$ shows equal advantage with $\mathrm{C}$ language and JAVA in terms of compatibility and design performance. Currently, $\mathrm{C}++$ language can be used together with WIN325DK, MFC, CLR, and NET, and it is the primary language of desktop program development. Although $\mathrm{C}++$ is thought as the improvement of $\mathrm{C}$ language, it is able to directly learn and apply $\mathrm{C}++$ language upon introduction. Besides, $\mathrm{C}++$ also has strong expansibility in terms of object orientation; it is different from $\mathrm{C}$ language, but it is also a brand-new programming language.

\section{Selection of programming language from perspective of practical application of software development}

\section{Selection principle of programming language}

Currently, in the fields of IT, enterprises, and people's life, the application of software can be seen everywhere, and the application function is in thousands of forms, thus the software development industry is absolutely a hot field in current IT circle. In the software development process, we shall follow its practical application purpose and functional features to select programming language. Firstly, we shall combine programming language with the definition, feature, basic principle, and function of developed software to carry out language design adaptation. When the code compiling is hard to be understood, the code can be re-compiled. For example, we can abandon parm+10. If we don't contact with later code, it is hard to understand why the code is abandoned, for its code is not wrong and the compiler function is not used; the code is a code which can be fully executed. Therefore, in the process of code compiling, we shall pay attention to structure echoing before and after code, that is, getting a knowledge of features of programming language; this is because it is able to reduce the work difficulty and work amount and improve work efficiency to great degree through deepening the understanding of programming language. 


\section{Selection of programming language according to overall structure}

The overall structure of developed software project is the key to determine how to select the programming language. Due to the fact that the software system consists of many parts, it is likely that many kinds of programming language will be applied in the development of one software, and the selection and language lies at the function of software project.

For example, in the process of developing web portals such as Wechat, Microblog or other applied communication software, we often apply PHP language which is the core of those software and webpage, and the operation data and processing of all website software can't be separated from this kind of dynamic technology. As a webpage script-based development language, its language style is inclined to popular C language and Perl language with high integration; it gathers all HTML webpage codes together and follows object-oriented principle to carry out design on webpage, desktop and other application program. Currently, in the popular Web2.0 sites, PHP language will be applied.

As a kind of open-source language, the structure feature of PHP shows good degree of maturity toward website and desktop software design. Through linking it with Linux, Apache HTTP Server, MySQL, and other procedure, it is able to form strong Linux operation system environment. Besides, PHP can be independently matched with Web server to carry out design of virtual software and form virtual host; this is also a virtual system environment promoted by most of data operators in China at present.

\section{Software development via most familiar programming language}

Currently, most of large-scale enterprises prefer to use JAVA and C language to carry out software development, and these two programming language can be actually applicable to software development. The main reason why the enterprises select these two kinds of language to carry out software development is that they can save time cost and economic cost. Besides, it is convenient to use them to carry out language innovation. For example, while developing some software which pays attention to repeated processing and manual labor, we shall flexibly select the language according to actual situation.

For example, in the process of game development, it is able to obtain extraordinary effect by use of multiple function of JAVA programming language to design the game script. Firstly, in one game software, we will make use of portability feature of JAVA to realize the purpose of enhancing overall effect of game. Furthermore, in recent years, with the continuous improvement of computer internet technology, some breakthroughs have been made in JAVA in the design of game software, which breaks the old mode of being limited to computer platform in the process of traditional software development; instead, the game design is transferred to mobile phone platform, and we start to design mobile game software.

Secondly, the reason why JAVA is often used by people is that it is familiar with the performance of various kinds of operation platform system. Due to the fact that the mainstream computer operation system is still Windows at present, people often apply JAVA language in the process of computer software development. This is because JAVA has a functional feature which C language and C++ language don't have. Except for the function of large-scale software development, JAVA can simplify the software compilation process, realize cross-platform transplantation, and make the software still reserve compiling environment after the software gets rid of fixed operation system platform so as to ensure the normal operation of program.

\section{Selection of development language according to software development environment}

The so-called software development depends on hardware environment which is the basis of software development. A kind of environment is matched with a kind of development language. For example, in the design of website script, we often apply PHP and Perl, for they are most mature script language at present; they have strong code library, low difficulty and can be conveniently applied; besides, they can clearly control the dynamic codes in webpage, thus the webpage collapse and other problems will not easily happen. However, in the military field, we generally select Ada language with high reliability, maintainability, real-time efficiency, and flexibility. It is born in United States 
Department of Defense and widely applied in the period of Gulf War, and it is a pure military programming language. Currently, in all American military software training center, Ada is one of necessary software programming tools. Its features include precise structure and multiple type, and it will carry out equivalent constraint, precision constraint, discriminant constraint, and subscribed constraint on each operand. In the compilation process, Ada language is good at inserting and checking code to change type of language and reduce the difficulty of language programming work. Meanwhile, Ada can identify the heavy-load concept of identifier and budget symbol, and this design concept provides great help for safe protection of military information ${ }^{[2]}$.

\section{Selection of programming language from perspective of development course of software development}

From large-size computer to small-size private computer, from low level to high level, the development language of computer software undergoes a process from greenness to maturity. Faced toward different software development condition, the computer programming language reflects its different ability.

\section{Selection of process-oriented software development language}

The process-oriented software development mainly pays attention to constitutive property, modularization, and functional abstraction of computer software. This paper thinks that it is required to select software development language according to the actual process orientation. How to select includes following two features.

1). The software program shall realize modularization. The modularization is an abstract expression of software function. Due to the fact that the module in computer hardware exists independently, each module has independent processing function, such as inputting and outputting of program code.

2). In the process of selecting process-oriented development language, we shall regard each process as an independent entity. In this way, it is able to clearly show processed result toward the users; furthermore, when the data code is input, all displayed results will show the consistency.

\section{Selection of aspect-oriented program development design language}

In the process of selecting programming language for aspect-oriented computer software development, we shall take the aspect as entry point, and this design is made according to users of object-oriented language. For example, in the process of software development, we carry out programming for the given system, take business logics as foothold, take software safety, persistent storage and logics as starting point rather than take the object as foothold to carry out software development. From perspective of definition relation of original program, through realization of aspect recombination, it is able to construct the link of various modules in software program and realize a complete software program making process. Meanwhile, it is required to pay attention to introducing and informing entry point and linkage point in terms of the definition of "aspect" as for aspect-oriented language so as to realize various links and demands between aspect mechanisms and realize the optimization on design software ${ }^{[3]}$.

\section{Conclusion}

Today, with rapid development of science and technology, each kind of development language has its existence value and position, and also has certain independence. In the process of software development, people will consider the unique practicability of those software, define their abstract level, simplify their internal structure, and then realize the high adaptability to the developed software and high efficiency of software development. Currently, the computer software and programming language increases at a dramatic rate, and people can realize more efficient and constructive software development through continuously absorbing new knowledge and carrying out deep research on various kinds of programming language. 


\section{References}

[1] Tian Zhi: Analysis on JAVA Programming Language Based on Computer Software Development, Silicon Valley, 2014(19):59-59.

[2] Hao Xu: Influence of Different Programming Language on Computer Application Software Development , Guide of Sci-tech Magazine, 2014(2):187.

[3] Li Dapeng: Research on Computer Software Development Language, Computer CD Software and Applications, 2012(6):196-196. 